\title{
Influence of separate and mixed experimental designs on reaction times to two simple visual stimuli
}

\author{
Camila Bruder and Luiz Eduardo Ribeiro-do-Valle \\ Universidade de São Paulo, Brazil
}

\begin{abstract}
Testing contexts have been shown to critically influence experimental results in psychophysical studies. One of these contexts that show important modulation of the behavioral effects of different stimulatory conditions is the separate (blocked) or mixed presentation of these stimulatory conditions. The study presents evidence that the apparent discriminabilities of two target stimuli can change according to which of these two testing contexts is used. A cross inside a ring and a vertical line inside a ring were presented as go stimuli in a go/no-go reaction time task. In one experiment, each of these stimuli was presented to a different group of volunteers and in another experiment they were presented to the same group of volunteers, randomly mixed in the blocks of trials. Similar reaction times were obtained for the two stimuli in the first experiment, and different reaction times (faster for the cross) in the second experiment. The latter result indicates that the two stimuli have different discriminabilities from the no-go stimulus; the cross having greater discriminability. This difference is however masked, presumably by the adoption of specific compensatory attentional sets, in a separate testing context. Keywords: context effects; reaction time; discriminability; attentional strategy.
\end{abstract}

Received 11 November 2008; received in revised form 17 February 2009; accepted 14 April 2009. Available online 29 June 2009

\section{Introduction}

Results obtained in visual discrimination tasks can be importantly influenced by an interaction between bottomup (sensory, early-level) visual processing and top-down (higher level) processing. This fact has been often forgotten when interpreting these results.

Bottom-up processing is based on two important features of the visual system, namely the parallel processing of different attributes of the visual scene in progressively higher cortical areas and the competition between visual neurons for representation and control (Desimone \& Duncan, 1995). These features of visual organization result in the favoring of the processing of non-homogeneities in the visual scene, the so-called salient or pop-out stimuli. These stimuli can differ from their background in terms of luminance, shape, color or any other attribute. They are more easily detected or discriminated.

Camila Bruder and Luiz Eduardo Ribeiro-do-Valle, Departamento de Fisiologia e Biofísica, Instituto de Ciências Biomédicas, Universidade de São Paulo. Correspondence regarding this article should be directed to: Luiz E. Ribeiro-doValle Departamento de Fisiologia e Biofísica, Instituto de Ciências Biomédicas, Universidade de São Paulo. Av. Prof. Lineu Prestes n. 1524, Cidade Universitária, São Paulo, SP, Brazil, CEP 05508000. Phone: (55 11) 3091-7364. E-mail: ribeiro@icb.usp.br
Top-down processing usually favors task relevant information. One well-known top-down influence is visual attention, which has been shown to improve performance as measured by reaction time (Lupiáñez, Milliken, Solano, Weaver, \& Tipper, 2001; Posner, Snyder, \& Cavidson, 1980) and/or accuracy (Bashinski \& Bacharach, 1980; Carrasco, Williams, \& Yeshurun, 2002; Muller \& Findlay, 1987). The specific demands of the task determine the adopted attentional set by which stimuli relevant to the task or stimuli which have some feature in common with them are attended to (Arnott, Pratt, Shore, \& Alain, 2001; Folk \& Remington, 1998; Folk, Remington, \& Johnston, 1992; Folk, Remington, \& Wright, 1994; Gibson \& Kelsey, 1998; Lambert, Wells, \& Kean, 2003; Remington, Folk, \& McLean, 2001; see Ruz \& Lupiáñez, 2002; see also Van der Heijdn, 1992). For instance, when someone is looking for a friend dressed in red among a crowd, this friend or people wearing red are more easily noticed than other people. There is a contingent orientation of attention as Folk and colleagues (Folk \& Remington, 1998; Folk, Remington, \& Johnston, 1992; Folk, Remington, \& Wright, 1994) called the phenomenon.

Another known top-down influence on task performance is the setting of a response criterion, which determines how much the observer is inclined to respond affirmatively to the target stimuli (Ciaramitaro, Cameron, \& Glimcher, 1999; MacMillam \& Creelmas, 
2005). It would occur at the decision stage of behavior control. It has been shown that changes in the difficulty of the task induce corresponding changes in the response criterion (Brown \& Steyvers, 2005).

The unexpected changes in performance often observed after small and apparently innocuous modifications in the experimental context should be ascribed mainly to the interaction between the influences mentioned above, considering that other potentially important influences have been properly controlled.

Psychophysical studies with human observers have provided many examples of contextual effects, such as the sequential effects (i.e., influences from previous trials on the current trial) observed in tasks ranging from visual discrimination (Scerif, Worden, Davidson, Seiger, \& Casey, 2006) to strategic decision making (Vlaev \& Chater, 2006), as well as the many types of effects reported by memory researchers, such as word frequency (Bodner \& Lindsay, 2003; McCabe \& Balota, 2007; Tulving \& Pearlstone, 1966). Other examples of contextual effects can be found in studies aiming at different goals, using different methodologies: e.g., Musseler and Kerzel (2004), with the Frölich effect; Kapoula, Yang, Coubard, Daunys and Orssaud, (2005), with different rates of transcranial magnetic stimulation within a block.

An important contextual influence on the outcome of an experiment is whether the testing conditions are distributed in separate blocks of trials or mixed in the same one. There are several reports in the literature indicating that very different results can be obtained with the two designs.

Investigating the influence of word-emotion congruency on auditory asymmetries, Techentin and Voyer (2007) obtained different results according to the experimental design adopted. They compared a blocked design (with the detection of a word target or of an emotional target in different blocks of trials) with a randomized design (in which the target was changed across trials) and found certain interferences of congruency only in the randomized designed. They argued that such interferences do not occur in the blocked design because of the establishment of an attentional set that avoids congruency effects.

The difference between separate and random designs has also been examined by researchers studying the temporal orientation of attention. For instance, subjects in a study by Correa, Lupiáñez, Milliken and Tudela (2004) were able to benefit from the use of a temporal visual cue that instructed them to prepare for the occurrence of a visual target (either early or late) only in a blocked design (i.e., when only one type of cue was used throughout the trials of the entire block), but not with a random design (i.e., when both types of cue could appear randomly in all the trials of the block).

Similarly, Faria and Machado-Pinheiro (2004) obtained shorter reaction times to a visual target stimulus after an auditory warning stimulus, when each one of the three warning stimulus-target stimulus onset asynchronies used occurred in separate blocks of trials but not when they occurred mixed in the same block of trials.

In another study, Machado-Pinheiro, Faria, Gawryszewski and Ribeiro-do-Valle (2004) found different reaction time effects of a visual onset cue, a visual offset cue and an auditory cue when these stimuli were presented randomly in the same blocks of trials, but not when they appeared in separate blocks of trials.

The same type of task design influence has been shown in reports by Azevedo, Squella and Ribeiro-doValle (2001) and by Macéa, Abbud, Lopes-de-Oliveira and Ribeiro-do-Valle (2006). They observed that the reaction time to a vertical line inside a ring in a go/nogo visual task did not differ from the reaction time to a cross inside a ring when these stimuli were presented in separate blocks of trials. However, when these stimuli were randomly presented in the same blocks of trials, the reaction time to the cross was shorter than that to the vertical line. These results were tentatively interpreted as due to the cross being easier to discriminate from the small ring than the vertical line (to be referred to in this paper as the cross having a higher discriminability than the line). In the blocked design, this difference between the discriminability of the line and that of the cross would have been masked, presumably by the adoption of specific attentional sets to deal with these stimuli, namely, a more intense mobilization of attention to deal with the line and a less intense mobilization of attention to deal with the cross. The adoption of any specific attentional set could obviously not occur in the mixed design, where participants did not know in advance whether the vertical line or the cross would appear next, and as a consequence the different discriminabilities of the two stimuli would be behaviorally expressed.

The results described immediately above were observed in both the first and second testing sessions of the experiments. In the second session, however, a peripheral prime stimulus appeared $100 \mathrm{~ms}$ before the target. In addition, the vertical line and the cross were presented to the same individuals in the experiment that used the blocked design. It is not clear how much these procedural details contributed to the results. It is of interest to verify whether similar results can be obtained for the second testing session in the absence of the peripheral prime stimulus and when the vertical line and the cross are presented to different groups of individuals.

Confirmation of these findings seems particularly important, as an adjustment of the criterion for responding would also be an appropriate strategy to deal with target stimuli of distinct discriminabilities. In this case, however, inverted results would be expected in the two experiments, because the criterion would be higher for the vertical line than for the cross in the blocked design and would be the same for these two stimuli in the mixed design. 
In this work, two experiments were designed in which volunteers were tested in a go/no-go reaction time task. The vertical line and the cross served as go stimuli and the small ring, as the no-go stimulus. In Experiment 1 , each of these go stimuli was presented to a different group of individuals. In Experiment 2, both go stimuli were presented to the same individuals, randomly mixed in the blocks of trials. Two testing sessions were run in each experiment. In both sessions, the reaction time did not differ between the vertical line and the cross in Experiment 1, but was shorter for the cross than for the vertical line in Experiment 2.

\section{Experiment 1}

In this experiment, two groups of volunteers performed a reaction time task in which they had to respond either to the line or to the cross and should not respond to the small ring. Considering previous evidence from our laboratory (Macéa et al., 2006), similar reaction times were expected for the line and the cross in the first testing session and, possibly, the second as well.

\section{Method}

\section{Participants}

Twelve young female adults, undergraduate or graduate students from the São Paulo University (mean age \pm sd: $21.6 \pm 0.57$ years-old) voluntarily took part in this experiment. All were right handed according to the Edinburgh Inventory (Oldfield, 1971) and had normal or corrected-to-normal vision. None of them had previous experience with psychophysical tasks or were aware of the purpose of the study. Written informed consent was obtained from all participants. This study was approved by the Ethics Committee of the Biomedical Sciences Institute (Instituto de Ciências Biomédicas - ICB, São Paulo University).

\section{Apparatus}

The volunteers were tested in a dimly illuminated (about $0.1 \mathrm{~cd} / \mathrm{m}^{2}$ ) and sound-attenuated room. Their heads were spatially positioned by a chin-and-front rest so that their eyes were $57 \mathrm{~cm}$ away from a 17inch video monitor. The background luminance of this screen was less than $0.01 \mathrm{~cd} / \mathrm{m}^{2}$. A central white 0.05 -degree wide square (fixation point, FP) and peripheral visual stimuli were presented on this screen. The volunteers were instructed to keep their eyes on the FP and to respond to some of these peripheral stimuli by pressing a key with their right index finger. An IBM-compatible computer controlled by programs developed with the MEL2 software (Psychology Software Tools Inc., Pittsburgh, PA, USA) generated the stimuli and recorded the responses.

\section{Procedure}

Each volunteer participated in two testing sessions on different days, not more than seven days apart. Before each session, the volunteer received a brief written explanation regarding the test. A more detailed explanation was given in the testing room while the volunteer performed some trial examples. The volunteer was then asked to perform about 20 practice trials.

Both testing sessions were identical. They consisted of four blocks of 64 trials each. Between one block and the next there was a short rest interval. Each trial began with the appearance of the FP. After a random interval of between 1850 and 2350 $\mathrm{ms}$, a target stimulus appeared. For one group of six volunteers, the targets were a vertical line (.96-degree long and 0.10-degree wide) inside a ring (1.72 degree in diameter and a .05-degree wide margin) and a small ring (0.29-degree in diameter and a 0.05 -degree wide margin) inside the ring. For another group of six volunteers, the targets were a cross (0.96-degree long and 0.05 -degree wide) inside the ring and the small ring inside the ring. All these stimuli were white, had the same luminance of $25.8 \mathrm{~cd} / \mathrm{m}^{2}$ and lasted $50 \mathrm{~ms}$. They could occur in any one of the four corners of a virtual square centered on the FP, 8 degree from this FP (see Figure 1.) The volunteer was instructed to respond as fast as possible to the "vertical line inside the ring" or to the "cross inside the ring" (the go stimuli) and not to respond to the "small ring inside the ring" (the no-go stimulus). The trial ended with a message lasting $200 \mathrm{~ms}$ at the site of fixation. Reaction time in milliseconds appeared when the volunteer responded between 150 and $600 \mathrm{~ms}$ after the onset of the go stimulus. The message "anticipated" or "slow" was displayed when the volunteer emitted a response before $150 \mathrm{~ms}$ after the onset of the go or no-go stimuli and did not respond until $600 \mathrm{~ms}$ after the onset of go stimuli, respectively. The message "incorrect" was displayed when the volunteer responded between 150 and $600 \mathrm{~ms}$ after the onset of the no-go stimulus. Error trials were repeated.

In each block, there were four sets of trials. In each set, eight trials consisted of the presentation of the go stimulus and eight trials consisted of the presentation of the no-go stimulus; these trials occurred randomly.

\section{Data analysis}

The mean of the four blocks median reaction time for each condition for each session was calculated for each volunteer. The total numbers of anticipated responses, slow responses and commission responses (false alarms) in each session were also evaluated for each condition for each volunteer. Reaction time data were treated by repeated measures analysis of variance (ANOVA) and the post hoc Newman-Keuls test. Factors in the ANOVA were group (line, VL, or 


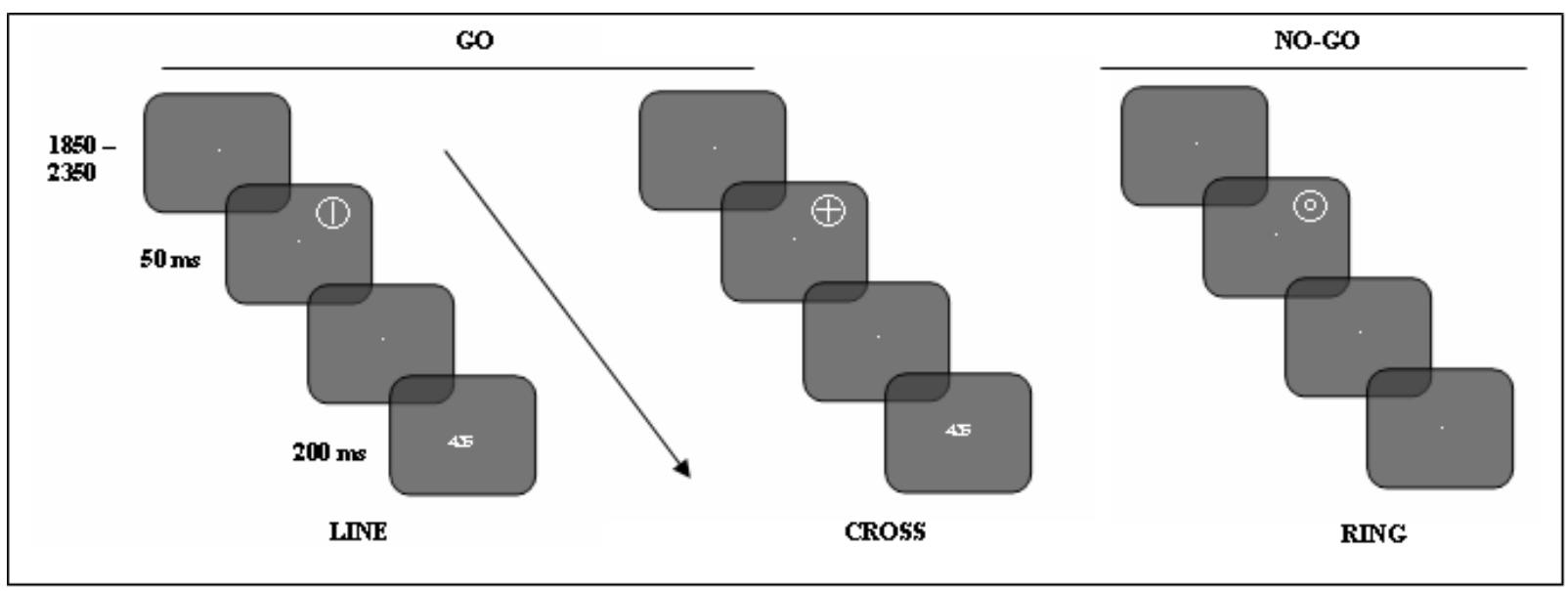

Figure 1. General design of Experiments 1 and 2. After a variable delay since the presentation of the fixation point, go stimuli (a vertical line inside a ring or a cross inside a ring) or the no-go stimulus (a small ring inside a ring) were presented for $50 \mathrm{~ms}$. Feedback information was given at the site of fixation.

cross, CR) and session (first or second). Accuracy data were treated by the Wilcoxon paired test. The significance level was set at .05.

\section{Results and Discussion}

No main effect of group $[\mathrm{F}(1,10)=0.067, \mathrm{p}=.800]$ or session $[F(1,10)=0.920, p=.359]$ was observed and no interaction occurred between these factors $[F(1,11)$ $=0.089, \mathrm{p}=.772]$. Reaction times for the line and cross were similar in both the first $(399 \pm 12 \mathrm{~ms}$ and $391 \pm 22$ $\mathrm{ms}$, respectively) and second session $(393 \pm 11 \mathrm{~ms}$ and $388 \pm 22 \mathrm{~ms}$, respectively) (Figure 2).

A separate ANOVA in which block was added as a third factor revealed a triple interaction $[\mathrm{F}(3,30)=3.14$, $\mathrm{p}=.04]$. The reaction time to the line was longer than that to the cross in the first block of the first testing session ( $\mathrm{p}$ $=.014$ ) but not in the three other blocks of this session neither in any of the four blocks of the second testing

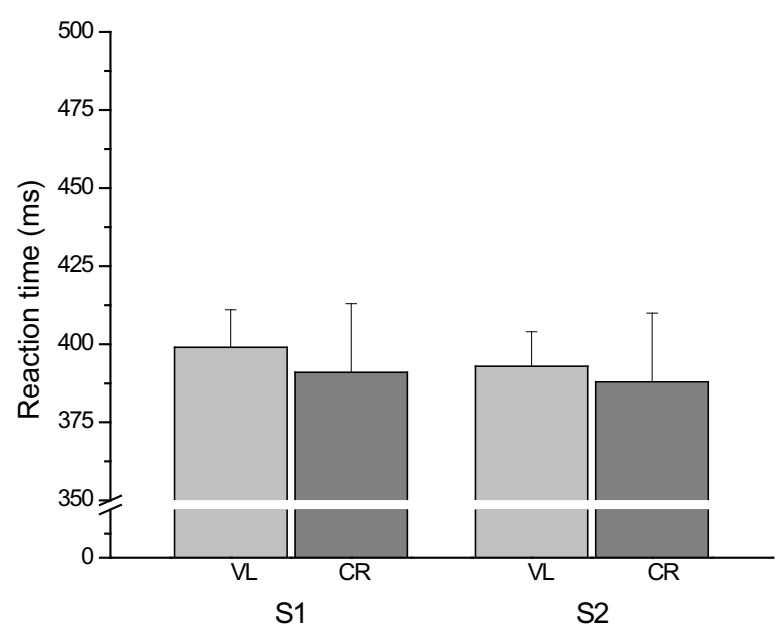

Figure 2. Mean reaction time $( \pm$ SEM) to the vertical line inside the ring (VL) and to the cross inside the ring (CR) in Experiment 1. S1, first session. $\mathrm{S} 2$, second session. $\mathrm{N}=6$. session. Reaction times to the line in the third and fourth blocks of the first testing session were shorter than that in the first block of this session (respectively, $p=.008$ and $p=.043$ ) [reaction times to the line in the second, third and fourth blocks of the second testing session were also shorter than that in the first block of the first testing session (respectively, $p=.042, p=.042$ and $p=.018$ )]

The analyses of errors showed no difference between the accuracy of responses for either group or session.

The overall absence of difference between reaction times to the line and the cross in both testing sessions of this experiment replicates and extends previous results obtained in our laboratory (Azevedo et al., 2001; Macéa et al., 2006). As already mentioned, similar reaction times to the two stimuli have been obtained in the first testing session of other experiments by our group. In the second testing session of these experiments, the same result was observed, but a peripheral visual prime stimulus was always presented before the line and the cross. The present results demonstrate that the occurrence or absence of the prime stimulus makes no difference.

It could be argued that the very small number of volunteers forming the two groups tested here casts some doubt on the reliability of the obtained results. If one considers, however, that exactly the same results were obtained for the first testing session in two previous studies of our laboratory (Azevedo et al., 2001; Macéa et al., 2006) in which 24 volunteers were used (12 in each study) and each one of these volunteers was tested with both stimuli (half of them in each study with the line first and the other half with the cross first, to control for order effects) these doubts should be alleviated.

The current findings are in agreement with the idea that specific attentional sets are adopted by the volunteers to deal with the line and the cross. More attention would be dedicated to processing the line than to processing the cross. This would have allowed these two stimuli to control the response in a similar way, despite their putative different discriminabilities. 


\section{Experiment 2}

In a previous study (Macéa et al., 2006), observation revealed that the line and the cross produce distinct reaction times when presented randomly in the same blocks of trials. In this experiment, an attempt to confirm and extend these results was made. We predicted that the reaction time to the cross would be shorter than that to the line, in both the first and second testing sessions, demonstrating that only when the same attentional set is used to deal with these stimuli does the higher discriminability of the cross become behaviorally expressed.

\section{Method}

\section{Participants}

Twelve female volunteers presenting the same characteristics described in Experiment 1 (mean age \pm sd: $21.8 \pm 0.44$ years old) were enrolled.

\section{Procedure}

The volunteers were tested as described in Experiment 1, with the main exception that the line and the cross appeared in the same block of trials. Each one of the four blocks of each testing session was formed by two sets of trials. In each set, eight trials consisted of the presentation of the line, eight trials consisted of the presentation of the cross and 16 trials consisted of the presentation of the small ring; these trials occurred randomly. Volunteers were instructed to respond to the line and to the cross, and not to respond to the small ring.

\section{Data analysis}

Reaction time data were analyzed as described in Experiment 1. For this experiment, the factors considered in the ANOVA were the session (first or second) and target (line or cross). Accuracy data were analyzed as described in Experiment 1.

\section{Results and Discussion}

Main effects of session $[\mathrm{F}(1,11)=13.849, \mathrm{p}=$ $.003]$ and target $[\mathrm{F}(1,11)=16.7, \mathrm{p}=.002]$ occurred, though no interaction occurred between these factors $[\mathrm{F}$ $(1,11)=.069, \mathrm{p}=.798]$.

Reaction times to the line were slower than those to the cross in both the first $(447 \pm 9 \mathrm{~ms}$ and $429 \pm 12 \mathrm{~ms}$, respectively) and second sessions (432 $\pm 9 \mathrm{~ms}$ and 413 $\pm 10 \mathrm{~ms}$, respectively). Reaction times were faster in the second session than in the first one for both the line and the cross (Figure 3).

A separate ANOVA in which block was added as a third factor revealed a block effect $[\mathrm{F}(3,33)=3.62, \mathrm{p}=$ $.023]$, in addition to the session and target effects already described. This analysis revealed also a session by block interaction $[\mathrm{F}(3,33)=4.40, \mathrm{p}=.010]$. Reaction times in the second, third and fourth blocks of the first testing session were shorter than that in the first block of this session (respectively, $\mathrm{p}=.037, \mathrm{p}=.002$ and $\mathrm{p}=.001$ ). The reaction time in the first block (and also those in the second, third and fourth blocks) of the second testing session was shorter than that in the first block of the first testing session $(\mathrm{p}=.001)$.

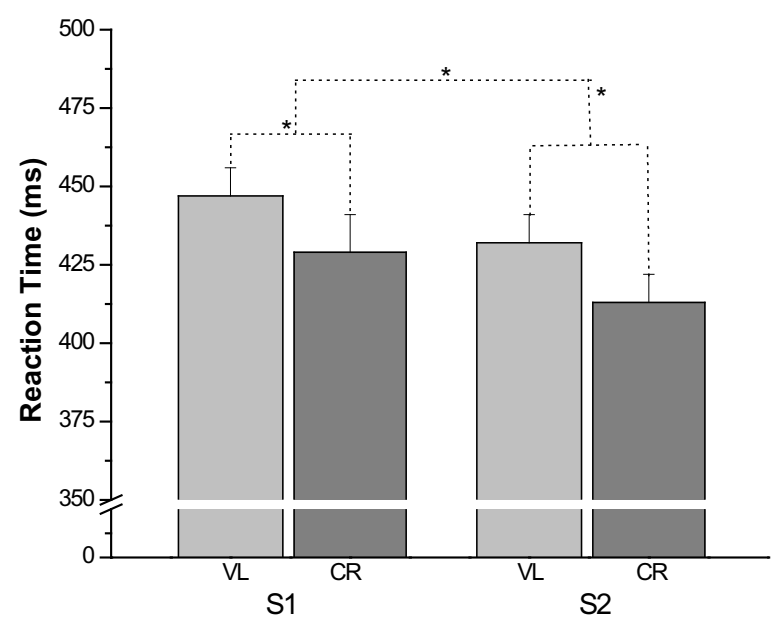

Figure 3. Mean reaction time $( \pm \mathrm{SEM})$ to the vertical line inside the ring (VL) and to the cross inside the ring (CR) in Experiment 2. $\mathrm{S} 1$, first session. S2, second session. Asterisks indicate significant differences. $\mathrm{N}=12$.

Another separate ANOVA on the difference between reaction times to the line and the cross having block and session as factors did not show any significant main effect or interaction.

In the first session, more omission errors occurred in trials with the line than in trials with the cross $(\mathrm{p}=$ .011). Fewer commission errors occurred in the second session than in the first session $(p=.017)$, which is probably a practice effect.

The use of a mixed context in the current experiment led to different results from those obtained in Experiment 1, namely, different reaction times to the line and the cross in both testing sessions. As the attentional set adopted by the volunteers should have been common for the line and the cross and their criterion for responding should also have been common for these stimuli, the faster reaction times and the lower omission rates for the cross strongly suggest that this stimulus is more easily discriminated from the small ring than the line is, in agreement with our initial supposition.

Considering that the number of volunteers tested in this experiment was not large, doubts about the reliability of the observed shorter reaction time to the cross than to the line could be raised. Perhaps this concern could be alleviated by the fact that at least for the first testing session exactly the same result was obtained with 12 other volunteers in a previous study of our laboratory (Macéa et al., 2006). 
It could also be questioned whether the advantage (of the order of $20 \mathrm{~ms}$ ) in the discrimination of the cross would have any biological meaning. Such advantage should be all the more important when the relative simplicity of the task is considered. In nature, where numerous similar discriminations must be performed in sequence, small differences like that could accrue, leading to important changes in the latency of behavioral responses.

More attention was probably paid to the task in the current experiment than in Experiment 1 as the task was more difficult because three stimuli, instead of two, had to be discriminated.

The most likely explanation for the reaction time decrease between the first testing session and the second testing session in the current experiment is that the volunteers could not completely master the task in the first testing session. A similar decrease was not observed in Experiment 1 probably because its task, which was easier (as indicated by the shorter reaction times obtained) than the one here, would have been completely mastered by the volunteers in the first testing session.

The absence of any changes in the difference between the reaction time to the line and to the cross across the blocks and across the sessions indicates that the relative responsivity of the different volunteers to these stimuli was quite stable.

\section{General Discussion}

In two experiments using a go/no-go reaction time task, the study showed different or equal reaction times to two target stimuli (a vertical line inside a ring or a cross inside a ring) depending on the testing context of these stimuli. When they were presented to different groups of volunteers, reaction times to them did not differ (Experiment 1). When they were presented to the same group of volunteers, mixed in the blocks of trials, reaction times were faster to the cross than to the line.

These results support the idea that these two stimuli present different discriminabilities with respect to the small ring, with the cross showing greater discriminability than the line. More importantly, they suggest that this difference can be compensated by the adoption of specific attentional sets, namely paying more attention to the task when the line is the go target and less attention when the cross is the go target, when this is viable, such as occurs in the separate testing context. Techentin and Voyer (2007) used a similar explanation to account for the reduction of the influence of congruency they observed when blocking their targets.

There are certainly other alternatives to explain the similar reaction times to the line and the cross in Experiment 1 than the suggested adoption by the volunteers of the strategy of paying more attention to the task when the line was the go target than when the cross was the go target. The most obvious ones are: a) that the volunteers tested with the cross paid less attention to the task (which would cause reaction time to the cross to increase), b) the volunteers tested with the line decreased their response criterion (which would cause reaction time to the line to decrease) or c) that the volunteers tested with the cross increased their response criterion (which would cause reaction time to the cross to increase). These alternatives seem however unlikely since they would imply, respectively, a) in the occurrence of more discrimination errors (false alarm responses) without any clear advantage, b) in the occurrence of more discrimination errors (false alarm responses) to the line and c) in an abnormal criterion change (inverse to task difficulty).

The more intense mobilization of attention when the line had to be discriminated from the small ring than when the cross had to be discriminated from the small ring, which was proposed to explain the results of Experiment 1, might not seem the most advantageous strategy to be adopted when having to deal with a more difficult task. Raising the response criterion would be better, since this strategy would, presumably, allow achievement of the same performance level and demand less mental effort. Possibly, the relatively short time available for responding would have devaluated this option. Indeed, by increasing the criterion to respond to the line, the $600 \mathrm{~ms}$ upper limit for responding in the trial could eventually be surpassed, leading to an error. Although this limit seems high enough to avoid errors due to a criterion increase, considering a mean reaction time of about $390 \mathrm{~ms}$, the organism might prefer to work on the safe side, considering that it does not know the exact moment when the interval available for responding ends.

Possible causes for the higher discriminability of the cross can only be speculated based on the evidence available so far. It may be due, for example, to its longer total border (approximately twice as much as that of the line). This would lead to a more intense stimulation of the retina.

Shevelev et al. (1998, 2007) described the activity of neurons in the cat primary visual cortex (V1) showing selectivity for cross-shaped figures. Such neurons increase (3.3-fold on average) their firing rate under stimulation by cruciform or corner figures flashing in their receptive fields, compared with single white light bars of preferred orientation, and exhibit smaller latencies when responding to cross-shaped figures than to light bars. Such cross-shaped figure-tuned neurons, which apparently extract second-order features at the early processing stage of V1, probably act in conjunction with the traditional first-order detectors, bar-tuned neurons. They might be involved in the relatively rapid identification of the cross demonstrated in the current study.

Adaptive behavior is critical for survival. It is attained by means of an interaction of numerous processes in a delicate and complex balance. Psychophysics can be a useful tool to elucidate some of these processes, but researchers must be always aware that uncontrolled processes might influence their results. For instance, we (Azevedo et al., 2001; Macéa et al., 2006) observed a 
robust attentional effect when we tested our volunteers with a peripheral prime stimulus and a go target stimulus represented by the vertical line inside the ring. However, we could not obtain any attentional effect when we used the cross inside the ring as the go target stimulus in the block of trials, everything else being the same. It is very likely that the different discriminabilities of the line and the cross, as demonstrated here, induced the adoption by the volunteers of specific attentional sets which affected the way they dealt with the prime stimulus.

In this paper we have investigated the influence of different experimental designs on psychophysical results. We demonstrated that the use of blocked and random contexts of presentation of two go stimuli (in this case a line and a cross inside a ring) leads to different results, probably because of the adoption by the observers of attentional sets specific for each of these contexts. This finding should be taken into account by researchers when planning their experiments and interpreting their results.

\section{References}

Arnott, S.R., Pratt, J., Shore, D.I., \& Alain, C. (2001). Attentional set modulates visual areas: an event-related potential study of attentional capture. Cognitive Brain Research, 12, 383-395.

Azevedo, E.L., Squella, S.A., \& Ribeiro-do-Valle, L.E. (2001). The early facilitatory effect of a peripheral spatially noninformative prime stimulus depends on target stimulus features. Brazilian Journal of Medical and Biological Research, 34, 803-813.

Bashinski, H.S., \& Bacharach, V.S. (1980). Enhancement of perceptual sensitivity as the result of selectively attending to spatial locations. Perception \& Psychophysics, 28, 241-248.

Bodner, G.E., \& Lindsay, D.S. (2003). Remembering and knowing in context. Journal of Memory and Language, 48, 563-580.

Brown, S., \& Steyvers, M. (2005). The dynamics of experimentally induced criterion shifts. Journal of Experimental Psychology: Learning, Memory, and Cognition, 31, 587-599.

Carrasco, M., Williams, P.E., \& Yeshurun, Y. (2002). Covert attention increases spatial resolution with or without masks: support for signal enhancement. Journal of Vision, 2, 467-479.

Ciaramitaro, V.M., Cameron, E.L., \& Glimcher, P.W. (1999). Stimulus probability directs spatial attention: an enhancement of sensitivity in humans and monkey. Vision Research, 41, 57-75.

Correa, A., Lupiáñez, J., Milliken, B., \& Tudela, P. (2004). Endogenous temporal orienting of attention in detection and discriminations tasks. Perception \& Psychophysics, 66, 264-278.

Desimone, R., \& Duncan, J. (1995). Neural mechanisms of selective attention. Annual Review of Neuroscience, 18, 193-222.

Faria Jr., A.J., \& Machado-Pinheiro, W. (2004). Looking for the GAP effect in manual responses and the role of contextual influences in reaction time experiments. Brazilian Journal of Medical and Biological Research, 37, 1175-1184.

Folk, C.L., \& Remington, R. (1998). Selectivity in distraction by irrelevant featural singletons: evidence for two forms of attentional capture. Journal of Experimental Psychology: Human Perception and Performance, 24, 847-858.

Folk, C.L., Remington, R.W., \& Johnston, J.C. (1992). Involuntary covert orienting is contingent on attentional control settings. Journal of Experimental Psychology: Human Perception and
Performance, 18, 1030-1044.

Folk, C., Remington, R.W., \& Wright, J. (1994). The structure of attentional control: contingent attentional capture by apparent motion, abrupt onset and color. Journal of Experimental Psychology: Human Perception and Performance, 20, 317-329.

Gibson, B.S., \& Kelsey, E.M. (1998). Stimulus-driven attentional capture is contingent on attentional set for displaywide visual features. Journal of Experimental Psychology: Human Perception and Performance, 24, 699-706.

Kapoula, Z., Yang, Q., Coubard, O., Daunys, G., \& Orssaud, C. (2005). Contextual influence of TMS on the latency of saccades and vergence. Neuroscience Letters, 376, 87-92.

Lambert, A., Wells, I., \& Kean, M. (2003). Do isoluminant color changes capture attention? Perception \& Psychophysics, 65, 495-507.

Lupiàñez, J., Milliken, B., Solano, C., Weaver, B., \& Tipper, S.P. (2001). On the strategic modulation of the time course of facilitation and inhibition of return. Quarterly Journal of Experimental Psychology $A, 54,753-773$.

Macéa, D.D., Abbud, G.A.C., Lopes-de-Oliveira, M., Fuga, N.B., \& Ribeiro-do-Valle, L.E. (2006). Control of attention by a peripheral visual cue depends on whether the target is difficult to discriminate. Brazilian Journal of Medical and Biological Research, 39, 957-968.

Machado-Pinheiro, W., Faria, A.J.P., Gawryszewski, L.G., \& Ribeirodo-Valle, L.E. (2004). Experimental context modulates warning signal effects. Brazilian Journal of Medical and Biological Research, 37, 1063-1069.

MacMillan, N.A., \& Creelman, C.D. (2005). Detection theory: A user's guide. Philadelphia: Lawrence Erlbaum Associates.

McCabe, D.P., \& Balota, D.A. (2007). Context effects on remembering and knowing: the expectancy heuristic. Journal of Experimental Psychology: Learning, Memory and Cognition, 33, 536-549.

Muller, H.J., \& Findlay, J.M. (1987). Sensitivity and criterion effects in the spatial cuing of visual attention. Perception \& Psychophysics, 42, 383-399.

Müsseler, J., \& Kerzel, D. (2004). The trial context determines adjusted localization of stimuli: reconciling the Frölich effect and onset repulsion effects. Vision Research, 44, 2201-2206.

Oldfield, R.C. (1971). The assessment and analysis of handedness: The Edinburgh Inventory. Neuropsychologia, 9, 97-113.

Posner, M.I., Snyder, C.R., \& Cavidson, B.J. (1980). Attention and the detection of signals. Journal of Experimental Psychology, 109, 160-174.

Remington, R.W., Folk, C.L., \& McLean, J.P. (2001). Contingent attentional capture or delayed allocation of attention? Perception \& Psychophysics, 63, 298-307.

Ruz, M., \& Lupiáñez, J. (2002). A review of attentional capture: on its automaticity

and sensitivity to endogenous control. Psicologica, 23, 283-309.

Scerif, G., Worden, M.S., Davidson, M., Seiger, L., \& Casey, B.J. (2006). Context modulates early stimulus processing when resolving stimulusresponse conflict. Journal of Cognitive Neuroscience, 18, 781-792.

Shevelev, I.A., \& Lazareva, N.A. (2007). Characteristics of responses of visual cortex neurons with sensitivity to bars or cross-shaped figures in cats. Neuroscience and Behavioral Physiology, 37, 311-319.

Shevelev, I.A., Lazareva, L.A., Sharaev, G.A., Novikova, R.V., \& Tikhomirov, A.S. (1998). Selective and invariant sensitivity to crosses and corners in cat striate neurons. Neuroscience, 84, 713-721.

Techentin, C., \& Voyer, D. (2007). Congruency, attentional set, and laterality effects with emotional words. Neuropsychology, 21, 646-655.

Tulving, E., \& Pearlstone, Z. (1966). Availability versus accessibility of information in memory for words. Journal of Verbal Learning and Verbal Behavior, 5, 381-386.

Van der Heijden, A.H.C. (1992). Selective Attention in Vision. London: Routledge.

Vlaev, I., \& Chater, N. (2006). Game relativity: how context influences strategic decision making. Journal of Experimental Psychology: Learning, Memory, and Cognition, 32, 131-149. 